\title{
Risk Factors for Treatment Discontinuation Caused by Adverse Events When Using Telaprevir, Peginterferon, and Ribavirin to Treat Chronic Hepatitis C: A Real-World Retrospective Cohort Study
}

\author{
Kazuki Ide, ${ }^{a, b, c}$ Yohei Kawasaki, ${ }^{*, a, c}$ Ryo Iketani, ${ }^{c}$ and Naohiko Masaki ${ }^{d}$ \\ ${ }^{a}$ Department of Pharmacoepidemiology, Graduate School of Medicine and Public Health, Kyoto University; Kyoto \\ 606-8501, Japan: ${ }^{b}$ Center for the Promotion of Interdisciplinary Education and Research, Kyoto University; Kyoto \\ 606-8501, Japan: ' Department of Drug Evaluation \& Informatics, Graduate School of Pharmaceutical Sciences, \\ University of Shizuoka; Shizuoka 422-8526, Japan: and ${ }^{d}$ The Research Center for Hepatitis and Immunology, \\ National Center for Global Health and Medicine; Chiba 272-8516, Japan. \\ Received December 2, 2016; accepted February 9, 2017; advance publication released online February 17, 2017
}

In this study, a nationwide database was used to identify the risk factors for treatment discontinuation due to adverse events during telaprevir, peginterferon, and ribavirin (T/PR) treatment, and estimate the increase in the occurrence of adverse events when patients have multiple risk factors at the same time. The risk factors were identified using univariate logistic regression analysis, and a Cochran-Armitage trend test was used to analyze the correlation between the number of risk factors and treatment discontinuation due to adverse events. Of the 25989 individuals registered in the database, 1668 (age, mean \pm standard deviation (S.D.): $58.0 \pm 9.9)$ were included in the study. Of these, $188(11.3 \%)$ discontinued T/PR therapy due to adverse events. In the univariate logistic regression analysis, sex, age, aspartate aminotransferase (AST) level, and platelet count were found to significantly affect the incidence of T/PR treatment discontinuation $(p<0.05)$. Furthermore, the incidence of treatment discontinuation gradually increased from 4.6 to $27.2 \%$ as the number of risk factors increased from 0 to 4 , and the Cochran-Armitage trend test showed a significant correlation $(p<0.001)$. In conclusion, this study not only revealed the risk factors for treatment discontinuation but also showed that patients with multiple risk factors are more likely to discontinue treatment due to adverse events compared to patients with fewer risk factors.

Key words hepatitis C; telaprevir; interferon; ribavirin; regional difference; nationwide database

\begin{abstract}
Hepatitis $\mathrm{C}$ is caused by hepatitis $\mathrm{C}$ virus (HCV) infection. Approximately 150 million individuals have chronic HCV infection worldwide, ${ }^{1)}$ and approximately 700000 individuals die from hepatitis C-related liver diseases every year. Treatments for chronic $\mathrm{HCV}$ infection that utilize direct-acting antiviral agents, such as sofosbuvir plus ribavirin or sofosbuvir plus ledipasvir, have been developed in recent years, and sustained virologic responses (SVRs) of $c a .90 \%$ have been achieved. ${ }^{2-4)}$ However, the cost of these treatments is high, and access to these treatments is limited. ${ }^{5,6)}$ As a result, common medical approaches are often still used to treat chronic HCV infection. Among the common approaches, a triple therapy comprising telaprevir, peginterferon, and ribavirin (T/PR) is still clinically applied for the treatment of genotype 1 hepatitis $\mathrm{C}$, but this treatment is more likely to cause adverse events than recent interferon-free treatments, which can make utilization of T/PR problematic. $^{7-9)}$ In the clinical trials of T/PR therapy, almost all of the participants experienced some adverse effects, with 10 to $20 \%$ of the patients having to discontinue the therapy as a result of these adverse events. ${ }^{7,8)}$ Therefore, the identification of risk factors for adverse events is important to ensure that patients can be treated as safely as possible.
\end{abstract}

A previous study suggested that low platelet count was a risk factor for hospitalization during T/PR therapy. ${ }^{10)}$ However, there are a limited number of studies focusing on the risk factors for adverse events during T/PR therapy, and patients with multiple risk factors have not been discussed in detail in previous clinical studies. ${ }^{10,11)}$ In clinical practice, patients typically have several functional disorders related to an un- derlying disease, and estimating the influence of multiple risk factors on adverse events is very important, because the risk of adverse events may increase in patients with multiple risk factors. ${ }^{12)}$ Therefore, detailed information based on real-world data is needed to help monitor patient status during therapy.

On the basis of this information, we conducted a real-world retrospective cohort study using a nationwide database to evaluate the risk factors for treatment discontinuation due to adverse events during T/PR therapy. In addition, we used the Cochran-Armitage trend test to estimate the increase in the occurrence of adverse events in patients with multiple risk factors.

\section{METHODS}

Study Design We conducted a real-world retrospective cohort study using a nationwide database containing information on hepatitis $\mathrm{C}$ treatment in Japan. ${ }^{13)}$ All of the 47 prefectures in Japan were invited to contribute to the database according to the Basic Act on Hepatitis Measures (Act No. 97; December 4, 2009), which was authorized by the Japanese government, and the database started to register cases in December 2009. Case registrations were performed according to the following process: 1) the physician filled out a standardized case report form, 2) the case report form was sent to the Hepatitis Information Center (Chiba, Japan), and 3) the case was registered in the database. The standardized case report form included the following information: sex, date of birth, age, clinical and/or histologic diagnosis, treatment experience, 
type(s) of treatment, treatment period (start and end of treatment), laboratory test results (serum HCV RNA level, HCV serotype and/or genotype, aspartate aminotransferase (AST) level, alanine aminotransferase (ALT) level, and platelet count), adverse events, date of completion of treatment, and treatment outcome. The SVR was used as a measure of the treatment outcome, and was determined using the serum HCV RNA level 24 weeks after cessation of treatment. If the serum HCV RNA level reduced to below the detectable level during this time, the case was judged to have an SVR.

Study Population The present study focused on patients with chronic hepatitis $C$ who were treated with T/PR, and analyzed the risk factors related to discontinuation of the treatment. The registration period was from December 2009 to August 2015, and patients with chronic hepatitis C were included in the study, unless they met one or more of the following exclusion criteria: they were infected with a $\mathrm{HCV}$ genotype other than genotype 1, they were also infected with hepatitis B virus (HBV), they suffered from cirrhosis, they were not treated with T/PR, they were under 16 years of age, the treatment was discontinued for a reason other than adverse events, or the required clinical data was missing. An age criterion was included because disease prognosis is different between children and adults. ${ }^{14)}$

Study Outcomes The primary outcome measure of this study was to estimate the influence of multiple risk factors on treatment discontinuation due to adverse events during T/PR therapy. The secondary outcome measures were 1) the cut-off values of the factors expressed as continuous variables, 2) the influence of each factor on treatment discontinuation, and 3) a descriptive summary of the adverse events that caused treatment discontinuation. The following categories are used to describe adverse events that caused treatment discontinuation: malaise, interstitial pneumonia, cerebral hemorrhage, anemia, anorexia, thrombocytopenia, psychoneurosis, retinopathy, and any other reason.

Statistical Analysis Continuous variables are presented as the mean \pm standard deviation (S.D.), and categorical variables are presented as a number and percentage (\%). The values for each variable (including age and laboratory test values) were taken immediately before T/PR therapy. The cut-off value for each factor (age, AST, ALT, platelet count) was determined using receiver operating characteristic (ROC) curve analysis, ${ }^{15)}$ and a univariate logistic regression analysis was performed to analyze the relationship between each factor and treatment discontinuation. Factors with $p<0.050$ in the univariate logistic regression analysis were then included in the multivariate analysis (a multivariate logistic regression analysis, and an analysis to estimate the influence of multiple risk factors on treatment discontinuation). For comparison, multivariate analyses were also performed with wider conditions, including factors with $p<0.150$ and $p<0.200$ in the univariate logistic regression analysis. The results of the univariate and multivariate logistic regression analyses were shown as odds ratios (ORs) and 95\% confidence intervals (CIs).

Before estimating the influence of multiple risk factors on treatment discontinuation, the number of risk factors and the incidence of treatment discontinuation were determined. Then, a Cochran-Armitage trend test was used to estimate the correlation between the number of risk factors and the incidence of treatment discontinuation due to adverse events. ${ }^{16,17)}$ In ad- dition, the Cox proportional hazard regression model was used to calculate the hazard ratio (HR) and 95\% CI of treatment discontinuation due to adverse events for each number of risk factors. ${ }^{18)}$

All of the statistical analyses were performed using SAS version 9.4 for Windows (SAS Institute Inc., Cary, NC, U.S.A.) and JMP Pro 13 (SAS Institute Inc.).

Ethical Considerations This study was conducted in accordance with the Declaration of Helsinki. Written informed consent was obtained from the participants prior to enrollment, and the protocol was approved by the Ethics Committee of the National Center for Global Health and Medicine of Japan on October 1st, 2009 (No. 738). The Strengthening the Reporting of Observational Studies in Epidemiology (STROBE) checklist used for this observational study is provided as Supplementary material (see Checklist 1).

\section{RESULTS}

Clinical Characteristics of the Study Subjects A flow diagram showing the study procedure is presented in Fig. 1, and the clinical characteristics of the study subjects are pre-

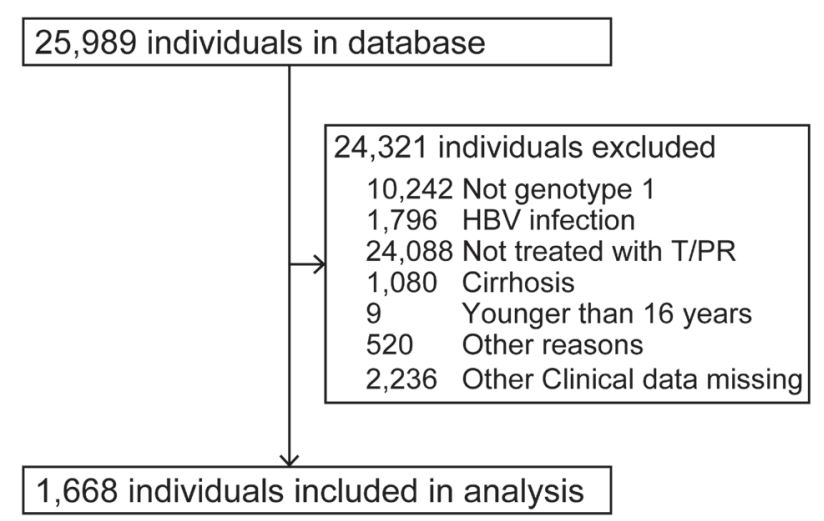

Fig. 1. Flow Diagram of the Study Procedure

Table 1. Clinical Characteristics

\begin{tabular}{lc}
\hline \multicolumn{1}{c}{ Characteristic } & T/PR $(N=1668)$ \\
\hline Age $(\mathrm{yr})$, mean \pm S.D. & $58.0 \pm 9.9$ \\
Sex, $N(\%)$ & \\
Male & $935(56.1)$ \\
Female & $733(43.9)$ \\
Platelet count $\left(\times 10^{4} / \mu\right.$ L), mean \pm S.D. & $16.4 \pm 5.3$ \\
AST $(\mathrm{IU} / \mathrm{L})$, mean \pm S.D. & $53.2 \pm 37.7$ \\
ALT $(\mathrm{IU} / \mathrm{L})$, mean \pm S.D. & $63.1 \pm 52.4$ \\
HCV viral load*, $N(\%)$ & \\
High & $1616(96.9)$ \\
Low & $52(3.1)$ \\
Treatment experience, $N(\%)$ & \\
Initial treatment & $787(47.2)$ \\
Retreatment & $881(52.8)$ \\
\hline
\end{tabular}

*High viral load: $\geq 5.0$ Log IU/mL (RT-PCR, Cobas ${ }^{\circledR}$ TaqMan HCV Test; Roche Molecular Systems, Pleasanton, CA, U.S.A.), or $\geq 100 \mathrm{KIU} / \mathrm{mL}$ (Cobas ${ }^{\circledR}$ Amplicor HCV Monitor v2.0; Roche Molecular Systems, Pleasanton, CA, U.S.A.); low viral load: $<5.0 \mathrm{Log} \mathrm{IU} / \mathrm{mL}$ (RT-PCR, Cobas ${ }^{\circledR}$ TaqMan HCV Test; Roche Molecular Systems, Pleasanton, CA, U.S.A.), or $<100 \mathrm{KIU} / \mathrm{mL}$ (Cobas ${ }^{\circledR}$ Amplicor HCV Monitor v2.0; Roche Molecular Systems, Pleasanton, CA, U.S.A.). ALT, alanine aminotransferase; AST, aspartate aminotransferase; HCV, hepatitis C virus; S.D., standard deviation; T/PR, telaprevir, peginterferon and ribavirin; yr, year. 
sented in Table 1. Of the 47 prefectures invited, 38 agreed to participate in the study. During the study period from December 2009 to August 2015, 25989 individuals were registered in the database. Among them, 1668 individuals (age, mean \pm S.D.: $58.0 \pm 9.9)$ were included in the study. The other 24321 individuals were excluded from the study because they met one or more of the exclusion criteria. The number of patients who met each exclusion criteria is shown in Fig. 1.

Treatment Discontinuation Due to Adverse Events Out of the 1668 patients included in the study, 188 (11.3\%) discontinued T/PR therapy due to adverse events. The categories of adverse event that caused treatment discontinuation are shown in Table 2. Several patients experienced two or more adverse events. Except for the "any other reason" category, thrombocytopenia, malaise, and anorexia were the main adverse events.

The cut-off values for the continuous variables estimated by ROC curve analysis were as follows: age, 62 years; AST level, $59 \mathrm{IU} / \mathrm{L}$; ALT level, $51 \mathrm{IU} / \mathrm{L}$; platelet count, $14.8 \times 10^{4} / \mu \mathrm{L}$. In the univariate logistic regression analysis, which included these and other categorical variables, sex (female: $\mathrm{OR}=1.675$; $95 \% \mathrm{CI}=1.234$ to $2.273 ; p<0.001)$, age $(\geq 62$ years: $\mathrm{OR}=2.330$; $95 \% \mathrm{CI}=1.706$ to $3.183 ; p<0.001)$, AST level $(\geq 59 \mathrm{IU} / \mathrm{L}$ : $\mathrm{OR}=1.478 ; 95 \% \mathrm{CI}=1.076$ to $2.031 ; p=0.016)$, and platelet count $\left(<14.8 \times 10^{4} / \mu \mathrm{L}: \quad \mathrm{OR}=1.584 ; 95 \% \quad \mathrm{CI}=1.168\right.$ to 2.147 ; $p=0.003)$ were found to significantly affect the incidence of $\mathrm{T} / \mathrm{PR}$ treatment discontinuation (Table 3). These variables were also found to significantly influence the incidence of treatment discontinuation due to adverse events during T/PR therapy in the multivariate analysis as follows: sex (female: $\mathrm{OR}=1.588 ; 95 \% \mathrm{CI}=1.164$ to $2.167 ; p=0.004)$, age ( $\geq 62$ years:
$\mathrm{OR}=2.219 ; 95 \% \mathrm{CI}=1.616$ to $3.048 ; p<0.001)$, AST level $(\geq 59 \mathrm{IU} / \mathrm{L}: \quad \mathrm{OR}=1.492 ; 95 \% \mathrm{CI}=1.073$ to $2.075 ; p=0.017)$, and platelet count $\left(<14.8 \times 10^{4} / \mu \mathrm{L}: \mathrm{OR}=1.399 ; 95 \% \mathrm{CI}=1.021\right.$ to $1.918 ; p=0.037$ ) (Table 3 ). The results of the univariate and multivariate analysis were exactly the same when using $p<0.150$ as the selection criterion for determining which factors would be included in the multivariate analysis. When using $p<0.200$ as the selection criterion, 5 potential risk factors (sex, age, AST, platelet count, and ALT) were selected in the univariate analysis, but the number of patients with all 5 potential risk factors was only 1 . As a result, we could not determine the statistical implications of this result.

Table 2. Incidences of Adverse Events That Led to Treatment Discontinuation

\begin{tabular}{lrc}
\hline \hline & \multicolumn{2}{c}{ T/PR } \\
\cline { 2 - 3 } & $N$ & $\%$ \\
\hline Total $N$ & 1668 & 100 \\
Treatment discontinuation* & 188 & 11.3 \\
Malaise & 51 & 3.1 \\
Interstitial pneumonia & 2 & 0.1 \\
Cerebral hemorrhage & 1 & 0.1 \\
Anemia & 29 & 1.7 \\
Anorexia & 42 & 2.5 \\
Thrombocytopenia & 52 & 3.1 \\
Psychoneurosis & 1 & 0.1 \\
Retinopathy & 10 & 0.6 \\
Any other reason & 118 & 7.1 \\
\hline
\end{tabular}

* Several patients had two or more adverse events that caused treatment discontinuation. T/PR, telaprevir, peginterferon and ribavirin.

Table 3. Univariate and Multivariate Logistic Regression Analysis

\begin{tabular}{|c|c|c|c|c|c|c|}
\hline & \multicolumn{2}{|c|}{$\begin{array}{c}\text { Treatment } \\
\text { discontinuation }\end{array}$} & \multicolumn{2}{|c|}{ Univariate logistic regression } & \multicolumn{2}{|c|}{ Multivariate logistic regression } \\
\hline & $N=1668$ & $N=188$ & OR $(95 \% \mathrm{CI})$ & $p$-value & OR $(95 \% \mathrm{CI})$ & $p$-value \\
\hline \multicolumn{7}{|l|}{ Sex } \\
\hline Male & 935 & 84 & 1 & & 1 & \\
\hline Female & 733 & 104 & $1.675(1.234,2.273)$ & $<0.001$ & $1.588(1.164,2.167)$ & 0.004 \\
\hline \multicolumn{7}{|l|}{ Age (yr) } \\
\hline$<62$ & 947 & 72 & 1 & & 1 & \\
\hline$\geq 62$ & 721 & 116 & $2.330(1.706,3.183)$ & $<0.001$ & $2.219(1.616,3.048)$ & $<0.001$ \\
\hline \multicolumn{7}{|l|}{ Treatment } \\
\hline Re-treatment & 881 & 94 & 1 & & - & \\
\hline Initial treatment & 787 & 94 & $1.136(0.838,1.538)$ & 0.412 & - & - \\
\hline \multicolumn{7}{|l|}{$\mathrm{HCV}$ VL* } \\
\hline Low & 52 & 5 & 1 & & - & \\
\hline High & 1616 & 183 & $1.200(0.471,3.057)$ & 0.702 & - & - \\
\hline \multicolumn{7}{|l|}{$\operatorname{AST}(\mathrm{IU} / \mathrm{L})$} \\
\hline$<59$ & 1182 & 119 & 1 & & 1 & \\
\hline$\geq 59$ & 489 & 69 & $1.478(1.076,2.031)$ & 0.016 & $1.492(1.073,2.075)$ & 0.017 \\
\hline \multicolumn{7}{|l|}{ ALT (IU/L) } \\
\hline$<51$ & 918 & 95 & 1 & & - & \\
\hline$\geq 51$ & 750 & 93 & $1.226(0.905,1.662)$ & 0.188 & - & - \\
\hline \multicolumn{7}{|c|}{ Platelet count $\left(\times 10^{4} / \mu \mathrm{L}\right)$} \\
\hline$\geq 14.8$ & 967 & 90 & 1 & & 1 & \\
\hline$<14.8$ & 701 & 98 & $1.584(1.168,2.147)$ & 0.003 & $1.399(1.021,1.918)$ & 0.037 \\
\hline
\end{tabular}

*High viral load: $\geq 5.0 \mathrm{Log}$ IU/mL (RT-PCR, Cobas ${ }^{\circledR}$ TaqMan HCV Test; Roche Molecular Systems, Pleasanton, CA, U.S.A.), or $\geq 100 \mathrm{KIU} / \mathrm{mL}$ (Cobas ${ }^{\circledR}$ Amplicor HCV Monitor v2.0; Roche Molecular Systems, Pleasanton, CA, U.S.A.); low viral load: $<5.0$ Log IU/mL (RT-PCR, Cobas ${ }^{\circledR}$ TaqMan HCV Test; Roche Molecular Systems, Pleasanton, CA, U.S.A.), or $<100 \mathrm{KIU} / \mathrm{mL}$ (Cobas ${ }^{\circledR}$ Amplicor HCV Monitor v2.0; Roche Molecular Systems, Pleasanton, CA, U.S.A.). ALT, alanine aminotransferase; AST, aspartate aminotransferase; $\mathrm{CI}$, confidence interval; $\mathrm{HCV}$, hepatitis $\mathrm{C}$ virus; $\mathrm{OR}$, odds ratio; VL, viral load; yr, year. 
Table 4. Number of Risk Factors and Cochran-Armitage Trend Test

\begin{tabular}{ccc}
\hline \hline Number of risk factors & $N$ & Treatment discontinuation, $N(\%)$ \\
\hline 0 & 264 & $12(4.6)$ \\
1 & 535 & $40(7.5)$ \\
2 & 560 & $77(13.8)$ \\
3 & 250 & $43(17.2)$ \\
4 & 59 & $16(27.2)$ \\
\hline Cochran-Armitage Trend Test & & $p$-value \\
& & $<0.001$ \\
\hline
\end{tabular}

Table 5. Cox Regression Analysis

\begin{tabular}{ccrc}
\hline \hline Risk factors & HR $(95 \% \mathrm{CI})$ & $p$-Value & Adjusted $p$-value \\
\hline 1 vs. 0 & $1.636(0.858,3.119)$ & 0.135 & 0.539 \\
2 vs. 0 & $3.074(1.672,5.649)$ & $<0.001$ & 0.001 \\
3 vs. 0 & $3.908(2.060,7.413)$ & $<0.001$ & $<0.001$ \\
4 vs. 0 & $6.621(3.130,14.003)$ & $<0.001$ & $<0.001$ \\
\hline
\end{tabular}

CI, confidence interval; HR, hazard ratio.

Multiple Risk Factors and Treatment Discontinuation The descriptive results for the number of risk factors and the incidence of treatment discontinuation are shown in Table 4. The incidence of treatment discontinuation gradually increased from 4.6 to $27.2 \%$, as the number of risk factors increased from 0 to 4 . The Cochran-Armitage trend test showed that there was a significant correlation between the number of risk factors and the incidence of treatment discontinuation due to adverse events $(p<0.001$, Table 4$)$. The Cox proportional hazard regression analysis also showed a significant increase in treatment discontinuation when patients had $\geq 2$ risk factors, and the point estimates of the adjusted HR ranged from 3.074 to 6.621 ( 2 vs. 0 : adjusted $\mathrm{HR}=3.074,95 \% \mathrm{CI}=1.672$ to 5.649, $p=0.001 ; 3$ vs. 0 : adjusted $\mathrm{HR}=3.908,95 \% \mathrm{CI}=2.060$ to 7.413, $p<0.001 ; 4$ vs. 0 : adjusted $\mathrm{HR}=6.621,95 \% \mathrm{CI}=3.130$ to $14.003, p<0.001)$ (Table 5).

\section{DISCUSSION}

We conducted a real-world retrospective cohort study to evaluate the risk factors for treatment discontinuation due to adverse events during T/PR therapy, and estimate the increase in treatment discontinuation due to adverse events when patients had multiple risk factors at the same time. During this study, we used a nationwide database including data from 38 prefectures $(80.9 \%$ of the prefectures in Japan), so the results would be representative of real-world treatment. ${ }^{13)}$ Our results revealed that several risk factors significantly influence the incidence of treatment discontinuation, and suggested that patients with multiple risk factors tended to exhibit a higher incidence of treatment discontinuation than that exhibited by patients with fewer risk factors.

Among the patients in this study, $11.3 \%$ discontinued T/PR therapy due to adverse events. The rate of treatment discontinuation due to adverse events was similar to that reported in previous clinical studies. ${ }^{7-9)}$ However, these previous clinical studies did not focus on the risk factors for treatment discontinuation due to adverse events. As has been reported regarding the efficacy and toxicity of PR therapy, ${ }^{19,20)}$ our study suggests that female patients, older patients, patients with high AST levels, and patients with low platelet count are significantly more likely to discontinue treatment due to adverse events. In addition, our results indicate that patients with a larger number of risk factors are more likely to discontinue treatment than are patients with fewer risk factors. The results of the Cochran-Armitage trend test were statistically significant, and the hazard ratio compared with patients with no risk factors increased from 1.636 to 6.621 as the number of risk factors increased from 1 to 4 . These results strongly suggest that the presence of multiple risk factors has a significant influence on the incidence of treatment discontinuation due to adverse events. Therefore, it is important to monitor these factors in patients with chronic hepatitis $\mathrm{C}$, to ensure that they can be treated safely.

The results of this study could be useful in ensuring that it is safe to utilize T/PR therapy for a particular patient, but this study also had several limitations. The main limitation of this study is the number of patients included in the analysis. After extracting the patients from the database and applying the exclusion criteria, $<2000$ patients remained that could be included in the analysis. Furthermore, the database only covered ca. $20 \%$ of patients who received a subsidy from the Japanese government. Therefore, the estimated values in this study may only reflect a limited population in clinical practice. ${ }^{13)}$ However, the hazard ratio of $0 v s .4$ risk factors was $>5$, and this suggests that the presence of multiple risk factors is of medical importance. Future studies using a database that covers a larger number of chronic hepatitis C patients in Japan, as well as more modern treatments (e.g. interferon-free regimens using direct-acting antivirals) will reveal the clinical importance of risk factors more accurately. The methods used in this study can be easily applied to other treatment regimens, which will be an advantage for future investigations.

Previous reports have indicated that the dose of a drug is also important in determining the risk of adverse events. ${ }^{21-23}$ ) Compared to that of previous reports, a major advantage of this study is that the database used included a larger number of patients from all over Japan. However, the database did not include any information about the dose of drugs used. The selected population of this study is also a potential limitation. In this study, we only included patients with genotype $1 \mathrm{HCV}$ in the analysis. This criterion was chosen to control differences in treatment outcome between genotypes. However, $\mathrm{T} / \mathrm{PR}$ therapy is also used to treat genotype $2 \mathrm{HCV}$ infection in clinical practice. Therefore, additional studies including the dose of drugs used, and including patients with genotype $2 \mathrm{HCV}$ will provide more useful information to help treat chronic hepatitis $\mathrm{C}$ efficiently.

In summary, this study not only revealed that there are several risk factors for treatment discontinuation but also showed that patients with multiple risk factors are more likely to discontinue treatment due to adverse events than are patients with a smaller number of risk factors. The results of the present study and future studies on interferon-free regimens using the methods applied in this study will provide important information to treat patients with chronic hepatitis $\mathrm{C}$ more safely.

Acknowledgments This work was supported by a Grantin-Aid from the Ministry of Health, Labour and Welfare of 
Japan (Research on Hepatitis: 2009-2014) to NM. The authors would like to thank Ms. Mikako Kajio, Ms. Asako Horihara, and Ms. Maiko Akutagawa for their technical assistance with the data analysis. We would also like to acknowledge the great contribution of the 38 prefectural representatives and all of the medical staff members engaged in long-term interferon treatment and data collection.

Conflict of Interest The authors declare no conflict of interest.

Supplementary Materials The online version of this article contains supplementary materials.

\section{REFERENCES}

1) World Health Organization. "Hepatitis C. Fact sheet No. 164." 〈http://www.who.int/mediacentre/factsheets/fs164/en/〉, cited 28 July, 2016.

2) Gane EJ, Stedman CA, Hyland RH, Ding X, Svarovskaia E, Symonds WT, Hindes RG, Berrey MM. Nucleotide polymerase inhibitor sofosbuvir plus ribavirin for hepatitis C. N. Engl. J. Med., 368, 34-44 (2013).

3) Lawitz E, Poordad FF, Pang PS, Hyland RH, Ding X, Mo H, Symonds WT, McHutchison JG, Membreno FE. Sofosbuvir and ledipasvir fixed-dose combination with and without ribavirin in treatment-naive and previously treated patients with genotype 1 hepatitis C virus infection (LONESTAR): an open-label, randomised, phase 2 trial. Lancet, 383, 515-523 (2014).

4) Mizokami M, Yokosuka O, Takehara T, Sakamoto N, Korenaga M, Mochizuki H, Nakane K, Enomoto H, Ikeda F, Yanase M, Toyoda H, Genda T, Umemura T, Yatsuhashi $H$, Ide T, Toda $N$, Nirei $K$, Ueno Y, Nishigaki Y, Betular J, Gao B, Ishizaki A, Omote M, Mo HM, Garrison K, Pang PS, Knox SJ, Symonds WT, McHutchison JG, Izumi N, Omata M. Ledipasvir and sofosbuvir fixed-dose combination with and without ribavirin for 12 weeks in treatment-naïve and previously treated Japanese patients with genotype 1 hepatitis C: an open-label, randomised, phase 3 trial. Lancet Infect. Dis., 15, 645-653 (2015).

5) Canary LA, Klevens RM, Holmberg SD. Limited access to new hepatitis $\mathrm{C}$ virus treatment under state medicaid programs. Ann. Intern. Med., 163, 226-228 (2015).

6) Pawlotsky JM. New hepatitis C therapies: the toolbox, strategies, and challenges. Gastroenterology, 146, 1176-1192 (2014).

7) McHutchison JG, Everson GT, Gordon SC, Jacobson IM, Sulkowski M, Kauffman R, McNair L, Alam J, Muir AJ, PROVE1 Study Team. Telaprevir with peginterferon and ribavirin for chronic $\mathrm{HCV}$ genotype 1 infection. N. Engl. J. Med., 360, 1827-1838 (2009).

8) Hézode C, Forestier N, Dusheiko G, Ferenci P, Pol S, Goeser T, Bronowicki JP, Bourliére M, Gharakhanian S, Bengtsson L, McNair L, George S, Kieffer T, Kwong A, Kauffman RS, Alam J, Pawlotsky JM, Zeuzem S, PROVE2 Study Team. Telaprevir and peginterferon with or without ribavirin for chronic HCV infection. N. Engl. J. Med., 360, 1839-1850 (2009).

9) Kumada H, Toyota J, Okanoue T, Chayama K, Tsubouchi H, Hayashi N. Telaprevir with peginterferon and ribavirin for treatment-naive patients chronically infected with HCV of genotype 1 in Japan. $J$. Hepatol., 56, 78-84 (2012).

10) Maasoumy B, Port K, Markova AA, Serrano BC, Rogalska-Taranta
M, Sollik L, Mix C, Kirschner J, Manns MP, Wedemeyer H, Cornberg M. Eligibility and safety of triple therapy for hepatitis C: lessons learned from the first experience in a real world setting. PLoS ONE, 8, e55285 (2013).

11) Lawitz E, Rodriguez-Torres M, Muir AJ, Kieffer TL, McNair L, Khunvichai A, McHutchison JG. Antiviral effects and safety of telaprevir, peginterferon alfa-2a, and ribavirin for 28 days in hepatitis C patients. J. Hepatol., 49, 163-169 (2008).

12) Nallamothu BK, Hayward RA, Bates ER. Beyond the randomized clinical trial: the role of effectiveness studies in evaluating cardiovascular therapies. Circulation, 118, 1294-1303 (2008).

13) Masaki N, Yamagiwa Y, Shimbo T, Murata K, Korenaga M, Kanto T, Mizokami M, Prefectural members contributing to the Japanese Interferon Database. Regional disparities in interferon therapy for chronic hepatitis C in Japan: a nationwide retrospective cohort study. BMC Public Health, 15, 566 (2015).

14) González-Peralta RP, Kelly DA, Haber B, Molleston J, Murray KF, Jonas MM, Shelton M, Mieli-Vergani G, Lurie Y, Martin S, Lang T, Baczkowski A, Geffner M, Gupta S, Laughlin M, International Pediatric Hepatitis C Therapy Group. Interferon alfa-2b in combination with ribavirin for the treatment of chronic hepatitis $\mathrm{C}$ in children: efficacy, safety, and pharmacokinetics. Hepatology, 42, 1010-1018 (2005)

15) Metz CE. Basic principles of ROC analysis. Semin. Nucl. Med., 8, 283-298 (1978)

16) Sasieni PD. From genotypes to genes: doubling the sample size. Biometrics, 53, 1253-1261 (1997).

17) Sekine I, Segawa Y, Kubota K, Saeki T. Risk factors of chemotherapy-induced nausea and vomiting: index for personalized antiemetic prophylaxis. Cancer Sci., 104, 711-717 (2013).

18) Grambsch PM, Therneau TM. Proportional hazards tests and diagnostics based on weighted residuals. Biometrika, 81, 515-526 (1994).

19) Minami T, Kishikawa T, Sato M, Tateishi R, Yoshida H, Koike K. meta-Analysis: mortality and serious adverse events of peginterferon plus ribavirin therapy for chronic hepatitis C. J. Gastroenterol., 48, 254-268 (2013)

20) Ide K, Sato I, Imai T, Hawke P, Yamada H, Kawasaki Y, Masaki N. Comparison of the safety profiles of pegylated interferon alpha-2a and alpha-2b administered in combination with ribavirin for chronic hepatitis C infection: A real-world retrospective cohort study. Biol. Pharm. Bull., 39, 2060-2065 (2016).

21) Oze T, Hiramatsu N, Yakushijin T, Yamada R, Harada N, Morishita $\mathrm{N}$, Oshita M, Mita E, Ito T, Inui Y, Inada M, Tamura S, Yoshihara H, Imai Y, Kato M, Miyagi T, Yoshida Y, Tatsumi T, Kasahara A, Hayashi N, Takehara T. The real impact of telaprevir dosage on the antiviral and side effects of telaprevir, pegylated interferon and ribavirin therapy for chronic hepatitis C patients with HCV genotype 1. J. Viral Hepat., 22, 254-262 (2015).

22) Kawaguchi $Y$, Iwane $S$, Kumagai $T$, Yanagita $K$, Yasutake T, Ide Y, Otsuka T, Eguchi Y, Ozaki I, Akiyama T, Kawazoe S, Mizuta T. Efficacy and safety of telaprevir, pegylated interferon $\alpha-2 b$ and ribavirin triple therapy in Japanese patients infected with hepatitis C virus genotype 1b. Intern. Med., 54, 2551-2560 (2015).

23) Gaeta GB, Precone DF, Felaco FM, Bruno R, Spadaro A, Stornaiuolo G, Stanzione M, Ascione T, De Sena R, Campanone A, Filice G, Piccinino F. Premature discontinuation of interferon plus ribavirin for adverse effects: a multicentre survey in 'real world' patients with chronic hepatitis C. Aliment. Pharmacol. Ther., 16, 1633-1639 (2002). 